\title{
The impact of lung ultrasound on clinical-decision making across departments: a systematic review
}

\author{
Micah L. A. Heldeweg ${ }^{1,2^{*}}$ D, Lian Vermue ${ }^{1}$, Max Kant ${ }^{1}$, Michelle Brouwer ${ }^{1,2,3}$, Armand R. J. Girbes ${ }^{1}$, \\ Mark E. Haaksma ${ }^{1,2}$, Leo M. A. Heunks ${ }^{1}$, Amne Mousa ${ }^{1,2,3}$, Jasper M. Smit ${ }^{1,2}$, Thomas W. Smits ${ }^{1}$, \\ Frederique Paulus ${ }^{3}$, Johannes C. F. Ket ${ }^{6}$, Marcus J. Schultz ${ }^{3,4,5}$ and Pieter Roel Tuinman ${ }^{1,2}$
}

\begin{abstract}
Background: Lung ultrasound has established itself as an accurate diagnostic tool in different clinical settings. However, its effects on clinical-decision making are insufficiently described. This systematic review aims to investigate the impact of lung ultrasound, exclusively or as part of an integrated thoracic ultrasound examination, on clinical-decision making in different departments, especially the emergency department (ED), intensive care unit (ICU), and general ward (GW).

Methods: This systematic review was registered at PROSPERO (CRD42021242977). PubMed, EMBASE, and Web of Science were searched for original studies reporting changes in clinical-decision making (e.g. diagnosis, management, or therapy) after using lung ultrasound. Inclusion criteria were a recorded change of management (in percentage of cases) and with a clinical presentation to the ED, ICU, or GW. Studies were excluded if examinations were beyond the scope of thoracic ultrasound or to guide procedures. Mean changes with range (\%) in clinical-decision making were reported. Methodological data on lung ultrasound were also collected. Study quality was scored using the Newcastle-Ottawa scale.

Results: A total of 13 studies were included: five studies on the ED (546 patients), five studies on the ICU (504 patients), two studies on the GW (1150 patients), and one study across all three wards (41 patients). Lung ultrasound changed the diagnosis in mean 33\% (15-44\%) and 44\% (34-58\%) of patients in the ED and ICU, respectively. Lung ultrasound changed the management in mean 48\% (20-80\%), 42\% (30-68\%) and 48\% (48-48\%) of patients in the ED, in the ICU and in the GW, respectively. Changes in management were non-invasive in $92 \%$ and $51 \%$ of patients in the ED and ICU, respectively. Lung ultrasound methodology was heterogeneous across studies. Risk of bias was moderate to high in all studies.
\end{abstract}

Conclusions: Lung ultrasound, exclusively or as a part of thoracic ultrasound, has substantial impact on clinicaldecision making by changing diagnosis and management in the EDs, ICUs, and GWs. The current evidence level and methodological heterogeneity underline the necessity for well-designed trials and standardization of methodology.

Keywords: Ultrasonography, Lung, Chest, Management, Clinical-decision making

*Correspondence: m.heldeweg@amsterdamumc.nl

${ }^{1}$ Department of Intensive Care Medicine, Amsterdam University Medical Centers, location VUmc, Postbox 7507, 1007MB Amsterdam, The Netherlands

Full list of author information is available at the end of the article

\section{Background}

Lung ultrasound is a rapidly growing point-of-care diagnostic and monitoring modality in emergency departments (EDs), intensive care units (ICUs), and general wards (GWs). It is an increasingly common addition to 
standard physical examination and has been included in many specialist training programs [1-3]. Lung ultrasound's advantages are that it is non-invasive, easy to learn, and accurate in discriminating pulmonary pathology [4-6]. Moreover, integrating lung ultrasound with bedside cardiac and caval ultrasound expands its utility to comprehensively assess cardiopulmonary status [7]. Clinical use of lung ultrasound may therefore allow quicker arrival at correct diagnosis and management leading to improved patient outcomes.

Lung ultrasound's prompt emergence is also accompanied with several knowledge gaps. Previous literature has addressed issues such as methodological heterogeneity and reproducibility $[8,9]$. However, whether the implementation of lung ultrasound affects clinical-decision making remains largely unaddressed.

This systematic review investigates how often use of lung ultrasound, exclusively or as a part of thoracic ultrasound, leads to changes in clinical-decision making across departments, e.g. ED, ICU and GW.

\section{Methods}

This systematic review was registered at the International Prospective Register of Systematic Reviews (CRD42021242977). The Preferred Reporting Items for Systematic Reviews and Meta-analysis guidelines were followed to safeguard transparent and complete reporting of our review.

\section{Search strategy}

A comprehensive search of available literature on impact of lung ultrasound on clinical-decision making was built with the help of a medical librarian. Medical subject headings, keywords, and synonyms pertaining to lung, ultrasound, decision making, and patient management were used to search PubMed (Medline) up to March 19th 2021, as well as Embase and Web of Science up to April 6th 2021 (Additional file 1).

\section{Study selection and inclusion}

Inclusion criteria for studies were: (i) Original research in the English language reporting on changes in clinicaldecision making following lung ultrasound examination exclusively or as a part of thoracic ultrasound. Clinicaldecision making was defined as either a clinician's diagnosis, management, or therapy. At least a recorded change of management, the overarching term encompassing changes of therapy, changes of level of care, disposition, or consultations was required for inclusion; (ii) Patients in the ED, ICU (including medium care unit), or GW; (iii) General clinical presentations including hemodynamic or respiratory instability.
Exclusion criteria were: (i) Examinations beyond the scope of thoracic ultrasound (e.g. transcranial or abdominal); (ii) Ultrasound used solely for guiding procedures; (iii) Outpatient, prehospital, or rural patient presentations; (iv) Specific clinical presentations (e.g. only patients with pulmonary embolism). Title and abstract screening was conducted by two investigators (LV and $\mathrm{MK})$ and conflicts were resolved with help of a third investigator (MLAH).

\section{Data extraction}

Two investigators (MLAH and LV) extracted the following data from each study: (i) population; (ii) diagnostic and/or monitoring protocol; (iii) reported clinical-decision making change(s). Changes in therapy were further subdivided into non-invasive (pharmacological, fluids, ventilator settings, physiotherapy) and invasive (surgical procedure, start or stop mechanical ventilation, invasive diagnostic, or dialysis); (iv) methodological aspects (probe, lung ultrasound scoring system, training, interrater agreement).

\section{Data synthesis}

Studies were categorized according to setting (ED, ICU, or GW). Depending on the availability of data, the impact on clinical-decision was shown as a number and percentage (\%) of change in diagnosis, change in management, and change in therapy. Meta-analysis was considered inappropriate due to anticipated heterogeneity and lack of inferential statistics. As data is restricted to a limited number of studies, total changes across studies were presented as mean with reported range to facilitate interpretation. Other reported metrics on clinical-decision making were textually presented when appropriate. Although multiple changes of management can occur in one examination, when possible, the results were presented as relative number of examinations leading to changes to enable the calculation of a percentage.

Lung ultrasound's major paradigm shift occurred after a landmark April 2008 study [10]. Studies before this date had significantly different lung ultrasound methodologies. These studies primarily relied on limited lung ultrasound protocols, stricter patient selection, and external sonographers further removed from clinical decisions. These studies were not included in the aggregate, but discussed separately.

\section{Quality assessment}

The Newcastle-Ottawa scale for cohort studies was used to assess risk of bias. This quality assessment tool classifies an observational study's risk of bias with 0-9 stars, which was further subdivided as high (0-3 stars), moderate (4-6 stars), or low (7-9 stars) quality [11]. 


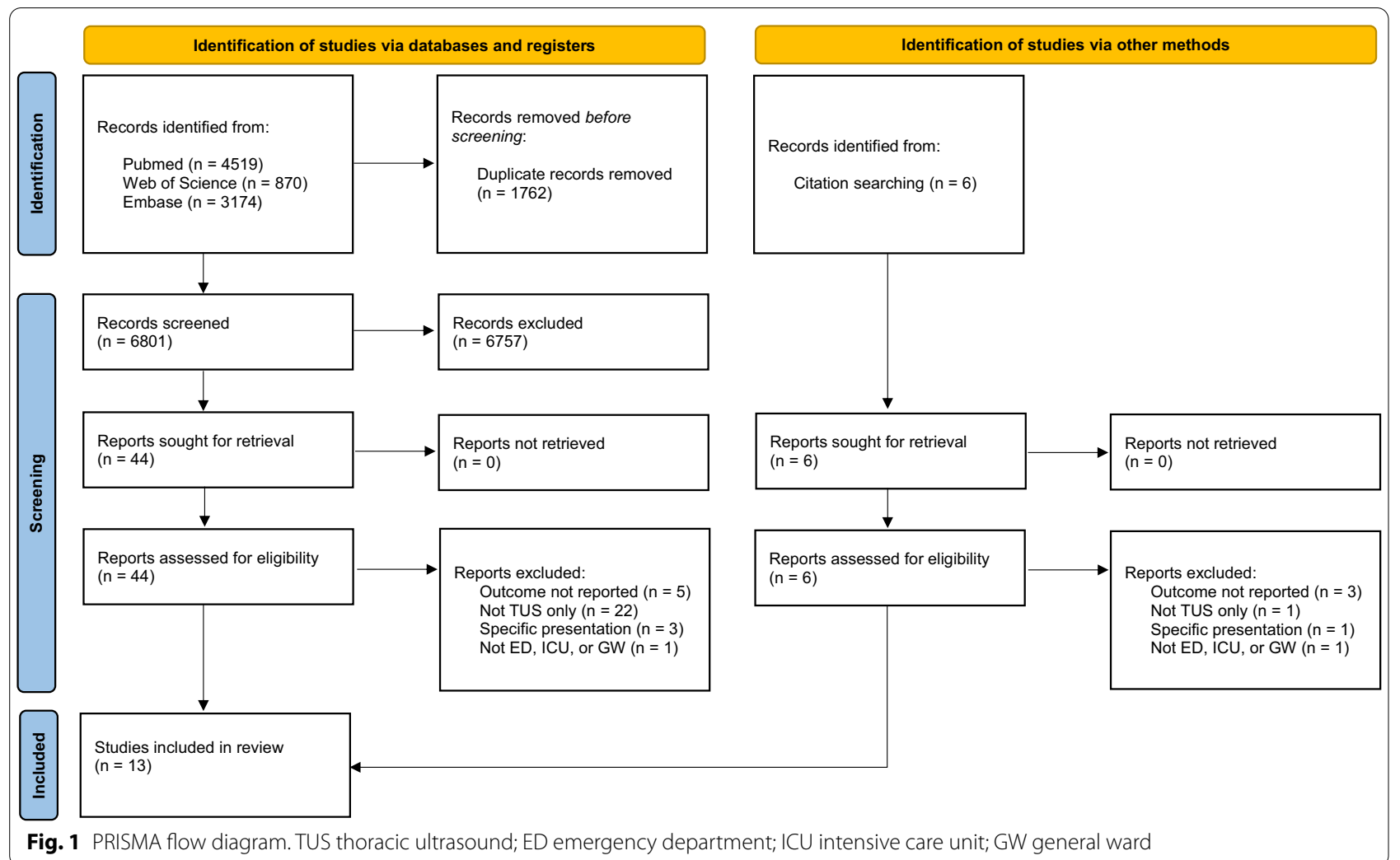

\section{Results}

A total of 8563 records were identified through the search in PubMed (53\%), Web of Science (10\%), and Embase (37\%). An additional six records were identified by screening reference lists. After removal of duplicates and screening using inclusion and exclusion criteria, 50 records were read in full. Ultimately, 13 studies with a total of 2142 patients were included for data extraction (Fig. 1).

All 13 studies had an observational design. Five studies were performed in the ED, five at the ICU, two at the $\mathrm{GW}$, and one at all three different departments. In the 10 studies performed after 2008 lung ultrasound was effectuated by the bedside clinician or investigators. In the three studies performed before April 2008, lung ultrasound was performed by independent radiologists or technicians. Five studies only investigated lung ultrasound, four included cardiac ultrasound, one included caval ultrasound, and one combined all thoracic ultrasound modalities.

\section{Effects of ultrasound on clinical-decision making in ED patients}

Outcomes of the five studies performed in the ED are presented in Table 1. Four studies investigated patients with dyspnea [12-15]. One study, performed in 2001, investigated patients with "acute chest symptoms" [16].

Changes in diagnosis, management, and therapy occurred in $33.2 \%$ (15.4-44\%), 47.6\% (19.6-80\%), and $41.2 \%$ (19.6-70\%), respectively. Of therapy changes $92 \%$ were non-invasive. Two studies included patients $<18$ years. One reported that changes in clinical impression (13.7\%) and changes in diagnosis (3.4\%) were less frequent in the pediatric population compared to adults (24.8\% of total population, mean age 3.4 years), whilst the other study did not specify any age-related characteristics $[12,13]$.

\section{Effects of ultrasound on clinical-decision making in ICU patients}

Outcomes of five studies performed in the ICU are presented in Table 2. Two studies evaluated patients with respiratory failure and two studies evaluated mechanically ventilated patients [17-20]. One ICU study, performed in 2008, evaluated the impact of "chest sonography" on all ICU patients during five months of on-demand chest radiography and five months of daily routine chest radiography [21].

Changes in diagnosis and management occurred in $43.8 \%(34-58 \%)$ and $42.2 \%(30-68 \%)$, respectively. Of therapy changes $51 \%$ were non-invasive. Rather than 
Table 1 Effect of ultrasound on clinical-decision making reported by ED studies

\begin{tabular}{|c|c|c|c|c|c|c|c|}
\hline Study & Year & $\begin{array}{l}\text { Patients }(\mathrm{n}) \text {, } \\
\text { symptom }\end{array}$ & Ultrasound & Diagnosis change & $\begin{array}{l}\text { Management } \\
\text { change }\end{array}$ & Therapy change & $\begin{array}{l}\text { Type of therapy } \\
\text { changes }\end{array}$ \\
\hline House & 2020 & 280, dyspnea & Lung & $124(44.3 \%)$ & $150(53.6 \%)$ & $125(44.6 \%)$ & $\begin{array}{l}\text { Invasive 9/125 } \\
\text { n-Invasive 116/125 }\end{array}$ \\
\hline Shah & 2016 & 117, dyspnea & Lung + cardiac & $18(15.4 \%)$ & $23(19.6 \%)$ & $23(19.6 \%)$ & $\begin{array}{l}\text { Invasive } 1 / 23 \\
\text { n-Invasive } 22 / 23\end{array}$ \\
\hline Russell & 2015 & 99, dyspnea & Lung + cardiac + caval & $17(17 \%)$ & $47(47 \%)$ & $42(42 \%)$ & $\begin{array}{l}\text { Invasive } 2 / 42 \\
\text { n-Invasive } 40 / 42\end{array}$ \\
\hline Goffi & 2013 & 50, dyspnea & Lung & $22(44 \%)$ & $40(80 \%)$ & $35(70 \%)$ & $\begin{array}{l}\text { Invasive 6/35 } \\
\text { n-Invasive 29/35 }\end{array}$ \\
\hline Total & & 546 & & $181(33.2 \%)$ & $260(47.6 \%)$ & 225 (41.2\%) & $\begin{array}{l}\text { Invasive 18/225 } \\
\text { n-invasive 207/225 }\end{array}$ \\
\hline Yuan & 2001 & $\begin{array}{l}78 \text { acute chest symp- } \\
\text { toms }\end{array}$ & Lung + cardiac & $52(66.7 \%)$ & $35(44.9 \%)$ & $34(43.6 \%)$ & $\begin{array}{l}\text { Invasive } 17 / 34 \\
\text { n-Invasive } 17 / 34\end{array}$ \\
\hline
\end{tabular}

TOTAL referred to the compilation of studies published after April 2008; ED emergency department

Table 2 Effect of ultrasound on clinical-decision making reported by ICU studies

\begin{tabular}{|c|c|c|c|c|c|c|}
\hline Study & Year & Patients (n), symptom & Ultrasound & Diagnosis change & Management change & Type of changes \\
\hline Barman & 2020 & 108, respiratory failure & Lung + cardiac & $40(37 \%)$ & $39(36 \%)$ & $\begin{array}{l}\text { Invasive 44/69 } \\
\text { n-Invasive 25/69 }\end{array}$ \\
\hline Haji & 2018 & 93, unspecified & Lung + cardiac & $53(58 \%)$ & $60(68 \%)$ & $\begin{array}{l}\text { Invasive } 2 / 60 \\
\text { n-Invasive 58/60 }\end{array}$ \\
\hline Wallbridge & 2017 & 50, respiratory failure & Lung + caval & $17(34 \%)$ & $15(30 \%)$ & $\begin{array}{l}\text { Invasive 1/15 } \\
\text { n-Invasive 14/15 }\end{array}$ \\
\hline Xirouchaki & 2014 & 253, MV adults & Lung & $N / A$ & $119(47 \%)$ & $\begin{array}{l}\text { Invasive 81/119 } \\
\text { n-Invasive 38/119 }\end{array}$ \\
\hline Total & & 504 & & $110(43.8 \%)$ & $233(42.2 \%)$ & $\begin{array}{l}\text { Invasive } 128 / 263 \\
\text { n-Invasive } 135 / 263\end{array}$ \\
\hline Kröner & 2008 & 36 adults & Lung & 16 of $43(37.2 \%)$ & 18 of $48(38 \%)$ & N/A \\
\hline
\end{tabular}

MV mechanically ventilated; N/A not available; TOTAL referred to the compilation of studies published after April 2008; ICU intensive care unit

reporting changes in diagnosis, one study reported the net reclassification index (85.6\%) as a metric for the impact of lung ultrasound on decision making [20]. According to one of the studies, patients who had multiple initial clinical diagnoses were more likely to have a change in management following ultrasound scanning (8/16 patients, compared to $7 / 34$ patients with a single diagnosis, $p=0.034$ ) [19]. None of the ICU studies differentiated between management or therapy changes.

\section{Effects of ultrasound on clinical-decision making in GW patients}

Two studies were performed on the GW with a mean change of management in $47.7 \%$ of examinations. Both studies did not report change in diagnosis or types of changes. On the Internal Medicine Ward, lung ultrasound led to a change in management in 25 out of $52(48.0 \%)$ examinations [22]. Pulmonologists used lung ultrasound across different wards to influence clinical-decision making, including treatment, in 548 (47.7\%) out of 1150 examinations [23].

A study from 1992, showed that chest sonographers' examinations on ED $(n=6)$, ICU $(n=19)$, and GW $(n=16)$ patients, assisted diagnosis in $27(66 \%)$ patients and changed management in $25(60.9 \%)$ of 41 patients [24].

\section{Lung ultrasound methodology}

Table 3 shows a large variance in lung ultrasound methodology across included studies. The most frequently used lung ultrasound protocol was 8-zone with a convex probe oriented perpendicular to the ribs.

\section{Quality assessment}

Table 4 shows the quality assessment. Four studies exhibit a high risk of bias, and nine studies a moderate risk of bias (4-6). None of the studies offer comparable control arms for clinical-decision making. 
Table 3 Lung ultrasound methodology of included studies

\begin{tabular}{|c|c|c|c|c|c|c|}
\hline Study & Zones & Orientation & B-line appraisal & Probe & Examiner & Interrater agreement \\
\hline \multicolumn{7}{|l|}{ ED } \\
\hline House 2020 & 10 & Perpendicular & $\begin{array}{l}\geq 2 \text { positive regions } \\
\text { with } \geq 3 \text { B-lines }\end{array}$ & Convex & Clinician + trained & $\begin{array}{l}\text { Experts: } 0.9 \\
\text { Clinician: } 0.8\end{array}$ \\
\hline Shah 2016 & 18 & Perpendicular & $\begin{array}{l}\geq 2 \text { positive regions } \\
\text { with } \geq 3 \text { B-lines }\end{array}$ & Phased & Clinician + trained & LVEF k:0.98 \\
\hline Russell 2015 & 8 & Perpendicular & $\begin{array}{l}\geq 2 \text { positive regions } \\
\text { with } \geq 4 \text { B-lines }\end{array}$ & Convex & Investigator + trained & Investigators K: 0.82 \\
\hline Goffi 2013 & 8 & Perpendicular & $\begin{array}{l}\geq 2 \text { positive regions } \\
\text { with } \geq 3 \text { B-lines }\end{array}$ & Convex & Investigator & N/A \\
\hline Yuan 2001 & N/A & N/A & N/A & $\begin{array}{l}\text { Linear + con- } \\
\text { vex + phased }\end{array}$ & $\begin{array}{l}\text { Technician } \\
+ \text { trained }\end{array}$ & N/A \\
\hline \multicolumn{7}{|l|}{$\mathrm{ICU}$} \\
\hline Barman 2020 & 8 & Parallel & $\begin{array}{l}\geq 2 \text { positive regions } \\
\text { with } \geq 3 \text { B-lines }\end{array}$ & Linear + convex & Investigator & $\mathrm{N} / \mathrm{A}$ \\
\hline $\begin{array}{l}\text { Haji } \\
2018\end{array}$ & 12 & Perpendicular & $\begin{array}{l}\geq 2 \text { positive regions } \\
\text { with } \geq 3 \text { B-lines }\end{array}$ & N/A & $\begin{array}{l}\text { Investigator + experi- } \\
\text { ence }\end{array}$ & k:0.69 \\
\hline Wallbridge 2017 & N/A & Parallel & $\begin{array}{l}\geq 2 \text { zones with B-lines: } \\
\text { diffuse }\end{array}$ & Convex + linear & Investigator + certified & N/A \\
\hline Xirouchaki 2013 & 12 & Perpendicular & $>1$ B-line in zone & Convex & $\begin{array}{l}\text { Investigator + experi- } \\
\text { ence }\end{array}$ & N/A \\
\hline Kröner 2008 & N/A & N/A & N/A & $\mathrm{N} / \mathrm{A}$ & Technician & $\mathrm{N} / \mathrm{A}$ \\
\hline \multicolumn{7}{|l|}{ GW } \\
\hline Mozzini 2016 & $28 / 8 / 2$ & Perpendicular & $\begin{array}{l}\geq 2 \text { positive regions } \\
\text { with } \geq 3 \text { B-lines }\end{array}$ & $\begin{array}{l}\text { Linear + con- } \\
\text { vex + phased }\end{array}$ & Clinicians + trained & Various \\
\hline Sferrazza papa 2016 & 8 & Perpendicular & $\begin{array}{l}\geq 2 \text { positive regions } \\
\text { with } \geq 3 \text { B-lines }\end{array}$ & Convex + linear & Clinicians + trained & N/A \\
\hline Yu 1992 & $\mathrm{~N} / \mathrm{A}$ & N/A & N/A & $\begin{array}{l}\text { Convex+lin- } \\
\text { ear + phased }\end{array}$ & Technician & $\mathrm{N} / \mathrm{A}$ \\
\hline
\end{tabular}

K kappa degree of agreement; N/A not available. The probes were grouped in major probe categories (e.g. phased, convex, linear) although their specific frequency range varied. The examiner was described as investigator, technician, or clinician. Training of examiners were grouped into experienced, trained and certified although the respective definition of the former varied substantially

\section{Discussion}

The main findings of this systematic review are: (i) Lung ultrasound resulted in a large proportion of diagnosis changes in the ED and ICU (34\% and 44\% of examinations, respectively); (ii) Lung ultrasound resulted in substantial management changes in the ED, ICU, and GW ( $48 \%, 42 \%$, and $48 \%$ of examinations, respectively); (iii) On the ED and ICU therapy changes were most frequently non-invasive (92\% and $51 \%$ respectively); (iv) Lung ultrasound methodology was heterogeneous across studies; v. Moderate to high risk of bias was present across all studies.

This study shows that bedside lung ultrasound is frequently a decisive tool in different clinical settings. This is an important finding: changes to physician behavior and subsequent modification of patient care might result in improvement in patient-centered outcomes. Moreover, even when no changes are effectuated, the confirmation of clinical impression could prevent uptake of further, costly or more invasive diagnostic and monitoring modalities. Additionally, the outcomes of the included studies were absolute (change versus no change), but ultrasound may also induce modification of prior likelihood. Resulting elimination of uncertainty may prevent delays of indicated care and, in some cases, patient harm $[25,26]$.

This study found that the majority of ultrasoundinduced changes were classified as non-invasive as opposed to invasive. This classification is limited in evaluating true effects of changes, both in diagnosis and management, on patient-centered outcomes. For example, a non-invasive change may be to abstain from increasing furosemide dose, but another may be to start rapid fluid resuscitation. At the same time, small changes in management should not be underestimated, as, for example, optimization of volume status may result in faster liberation from mechanical ventilation [27].

Consistent with previous literature, the majority of studies integrated lung ultrasound with other thoracic ultrasound modalities. This is a reasonable approach as pathologies encountered upon thoracic ultrasound are often (patho)physiologically linked. Findings on lung 
Table 4 Quality assessment of studies for this systematic review's outcome of interest using the Newcastle-Ottawa scale for cohort studies

\begin{tabular}{|c|c|c|c|c|c|c|c|c|c|}
\hline & \multicolumn{4}{|l|}{ Selection } & \multirow{2}{*}{$\begin{array}{l}\text { Comparability } \\
\text { Comparability } \\
\text { of cohorts on } \\
\text { the basis of } \\
\text { the design of } \\
\text { analysis }\end{array}$} & \multicolumn{3}{|l|}{ Outcomes } & \multirow[t]{2}{*}{ Total } \\
\hline & Representativeness & $\begin{array}{l}\text { Selection } \\
\text { of non- } \\
\text { exposed }\end{array}$ & $\begin{array}{l}\text { Ascertainment } \\
\text { of exposure }\end{array}$ & $\begin{array}{l}\text { Outcome } \\
\text { not } \\
\text { present } \\
\text { at start of } \\
\text { study }\end{array}$ & & $\begin{array}{l}\text { Assessment } \\
\text { of outcome }\end{array}$ & $\begin{array}{l}\text { Sufficient } \\
\text { follow up } \\
\text { time }\end{array}$ & $\begin{array}{l}\text { Adequacy } \\
\text { of follow } \\
\text { up of } \\
\text { cohorts }\end{array}$ & \\
\hline House 2020 & $\star$ & 谉 & $\star$ & $\star$ & 娄放 & $\star$ & $\star$ & $\star$ & $6 / 9$ \\
\hline Shah 2016 & $\star$ & 糸 & 论 & $\star$ & 类放 & $\star$ & $\star$ & $\star$ & $5 / 9$ \\
\hline Russel 2015 & $\star$ & 计 & $\star$ & $\star$ & 论虾 & $\star$ & $\star$ & $\star$ & $6 / 9$ \\
\hline Goffi 2013 & $\star$ & 拧 & it & $\star$ & 论动 & 类 & $\star$ & $\star$ & $4 / 9$ \\
\hline Yuan 2001 & $\star$ & 论 & it & $\star$ & 论动 & से & $\star$ & $\star$ & $4 / 9$ \\
\hline $\begin{array}{l}\text { Barman } \\
2020\end{array}$ & $\star$ & 谈 & से & मे & 出年 & मै & $\star$ & $\star$ & $3 / 9$ \\
\hline Haji 2018 & $\star$ & से & $\star$ & $\star$ & 论虾 & $\star$ & $\star$ & $\star$ & $6 / 9$ \\
\hline $\begin{array}{l}\text { Wallbridge } \\
2017\end{array}$ & $\star$ & 计 & it & $\star$ & 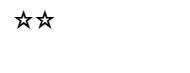 & th & $\star$ & $\star$ & $4 / 9$ \\
\hline $\begin{array}{l}\text { Xirouchaki } \\
2013\end{array}$ & $\star$ & 败 & से & 败 & 出年 & से & $\star$ & $\star$ & $3 / 9$ \\
\hline Kröner 2008 & $\star$ & 谈 & से & $\star$ & 论能 & $\star$ & $\star$ & $\star$ & $5 / 9$ \\
\hline $\begin{array}{l}\text { Mozzini } \\
2016\end{array}$ & $\star$ & 计 & मे & मे & 论虾 & 论 & $\star$ & $\star$ & $3 / 9$ \\
\hline $\begin{array}{l}\text { Sferrazza } \\
2016\end{array}$ & $\star$ & 谈 & $\star$ & $\star$ & 论败 & it & $\star$ & $\star$ & $5 / 9$ \\
\hline Yu 1992 & 计 & 计 & मे & $\star$ & 论㑔 & से & $\star$ & $\star$ & $3 / 9$ \\
\hline
\end{tabular}

Empty stars reflects lack of sufficient quality on the respective domains. Full starts reflect sufficient quality on respective domains where total represents high (0-3 stars), moderate (4-6 stars), or low (7-9 stars) risk of bias

ultrasound may support, modify, or moderate cardiac and caval ultrasound's findings and vice versa. Moreover, previous research also showed that an integrated thoracic ultrasound approach performs better than its individual components [28]. Other studies have expanded bedside ultrasound beyond thorax to include transcranial and abdominal ultrasound and found even higher impact on clinical management (60\% and 69\%, respectively) $[29,30]$. Evidently, point-of-care ultrasound modalities, combined or separately, have a substantial impact on clinical-decision making.

The current study examines the use of lung ultrasound in ED, ICU, and GW; three hospital departments where point-of-care ultrasound is very relevant. Results across these hospital settings were similar. Interestingly, the use of point-of-care ultrasound has expanded beyond hospital medicine. One study showed that the introduction of point-of-care ultrasound in general practice alters the diagnostic process and results in changes of diagnosis and management in half of patients [31]. Similarly, prehospital (and rural) studies employing a wide variety of POCUS examinations found a significant benefit that can dramatically alter disposition and treatment $(50 \%$ of patients) and correlated well with in-hospital diagnostic results $[32,33]$.
The methodology and quality assessment tables highlight weaknesses in current lung ultrasound research. Methodological inconsistencies are frequent amongst lung ultrasound investigations, may impact findings, and limit clinical reproducibility or generalizability $[8,9,34]$. The lack of comparator for any of the studies may be intrinsic to the selected outcome, but emphasizes the need for controlled and well-designed studies to study the effect of lung ultrasound beyond clinician behavior: patient-centered and hospital level outcomes. Even excellent diagnostic tools do not necessarily lead to improved patient-centered outcomes [35]. Similarly, wrongful interpretation of ultrasound accompanied by unwarranted change of management may have undesired effects. Currently, several trials are either underway or recently published that may potentially provide higher quality evidence [36-38].

This is the first study that systematically and exhaustively, including a total of 13 studies and 2142 patients, describes the impact of lung ultrasound on clinical-decision making. The search strategy was extensive (including three databases) to enhance identification of relevant studies. Strict methodology was used, including inclusion and exclusion criteria to increase homogeneity across studies, a recurring issue in ultrasound literature. 
A limitation to the current study is that the outcome of interest, physician behavior, is not necessarily associated with improvement of patient-centered outcomes. Assessment of the latter would require randomized or blinded studies to avoid confounding factors. Moreover, ultrasound-driven intended changes in physician behavior may not necessarily be executed. Feasibility studies are required to assess actual management effectuation. Furthermore, assessment of publication bias, e.g. by a funnel-plot, was not done. Lastly, it is possible that not all studies were identified due to the requirement of English language.

\section{Conclusions}

Lung ultrasound, exclusively or as a part of thoracic ultrasound, has a major impact on clinical-decision making by changing diagnosis management, and therapy in different clinical settings. However, the current evidence level and methodological heterogeneity underlines the invariable necessity for well-designed trials and standardization of ultrasound methodology.

\section{Abbreviations}

ED: Emergency Department; GW: General ward; ICU: Intensive Care Unit.

\section{Supplementary Information}

The online version contains supplementary material available at https://doi. org/10.1186/s13089-021-00253-3.

Additional file 1. a: First PubMed search 3575. b: Thorax added 944 to PubMed search. c: Embase search. d: Thorax added to Embase search. e: Web of Science search. f: Thorax added to Web of Science search.

\section{Acknowledgements}

Not applicable.

\section{Authors' contributions}

All authors (MLAH, LV, MK, MB, ARJG, MEH, LMAH, AM, JMS, TS, FP, JCFK, MJS, and PRT) were responsible for the conception of the work. MLAH, LV, MK, JCFK, and PRT were responsible for the design design of the work, as well as the acquisition and analysis of the data. MLAH, LV, and PT were responsible for the first draft of the manuscript. Subsequently all authors provided critical revisions for until the final manuscript was completed. All authors read and approved the final manuscript and ensured that questions related to the accuracy or integrity of any part of the work were investigated and resolved.

\section{Funding}

No funding was received for this study.

\section{Availability of data and materials}

The datasets used and/or analyzed during the current study are available from the corresponding author on reasonable request.

\section{Declarations}

Ethics approval and consent to participate Not applicable.
Consent for publication

Not applicable.

\section{Competing interests}

The authors declare that they have no competing (financial or non-financial) interests.

\section{Author details}

${ }^{1}$ Department of Intensive Care Medicine, Amsterdam University Medical Centers, location VUmc, Postbox 7507, 1007MB Amsterdam, The Netherlands. ${ }^{2}$ Amsterdam Leiden IC Echography (ALIFE), Amsterdam, The Netherlands.

${ }^{3}$ Department of Intensive Care, Amsterdam University Medical Centers, location AMC, Amsterdam, The Netherlands. ${ }^{4}$ Mahidol Oxford Tropical Medicine Research Unit (MORU), Mahidol University, Bangkok, Thailand. ${ }^{5}$ Nuffield Department of Medicine, University of Oxford, Oxford, UK. ${ }^{6}$ Medical Library, Amsterdam University Medical Centers, location VUmc, Amsterdam, The Netherlands.

Received: 27 July 2021 Accepted: 25 December 2021

Published online: 10 January 2022

\section{References}

1. Touw HRW, Tuinman PR, Gelissen HPMM et al (2015) Lung ultrasound: routine practice for the next generation of internists. Neth J Med 73:100-107

2. Solomon SD, Saldana F (2014) Point-of-care ultrasound in medical education-stop listening and look. N Engl J Med 370:1083-1085

3. Arts L, Lim EHT, van de Ven PM et al (2020) The diagnostic accuracy of lung auscultation in adult patients with acute pulmonary pathologies: a meta-analysis. Sci Rep 10:1-11

4. Winkler MH, Touw HR, van de Ven PM et al (2018) Diagnostic accuracy of chest radiograph, and when concomitantly studied lung ultrasound, in critically ill patients with respiratory symptoms: a systematic review and meta-analysis. Crit Care Med 46:e707-e714

5. Nooitgedacht J, Haaksma M, Touw HRW, Tuinman PR (2018) Perioperative care with an ultrasound device is as Michael Jordan with Scotty Pippen: At its best! J Thorac Dis 10:6436-6441

6. Orso D, Guglielmo N, Copetti R (2018) Lung ultrasound in diagnosing pneumonia in the emergency department: a systematic review and meta-analysis. Eur J Emerg Med 25:312-321

7. Mayo PH, Copetti R, Feller-Kopman D et al (2019) Thoracic ultrasonography: a narrative review. Intensive Care Med 45:1200-1211

8. Neuteboom OB, Heldeweg ML, Pisani L et al (2020) Assessing extravascular lung water in critically ill patients using lung ultrasound: a systematic review on methodological aspects in diagnostic accuracy studies. Ultrasound Med Biol 46(7):1557-1564

9. Haaksma ME, Smit JM, Heldeweg MLA et al (2020) Lung ultrasound and B-lines: B careful! Intensive Care Med 46(3):544-545

10. Lichtenstein DA, Mezière GA (2008) Relevance of lung ultrasound in the diagnosis of acute respiratory failure the BLUE protocol. Chest 134:117-125

11. Wells G, Shea B, O'Connell D, et al (1993) Newcastle-Ottawa scale (NOS) for assessing the quality of nonrandomised studies in meta-analyses. Available via OHRI. http://www.ohri.ca/programs/clinical_epidemiology/ oxford.asp. Accessed 22 July 2021

12. House DR, Amatya Y, Nti B, Russell FM (2020) Impact of bedside lung ultrasound on physician clinical decision-making in an emergency department in Nepal. Int J Emerg Med 13:14

13. Shah SP, Shah SP, Fils-Aime R et al (2016) Focused cardiopulmonary ultrasound for assessment of dyspnea in a resource-limited setting. Crit Ultrasound J 8:7

14. Russell FM, Ehrman RR, Cosby K et al (2015) Diagnosing acute heart failure in patients with undifferentiated dyspnea: a lung and cardiac ultrasound (LuCUS) protocol. Acad Emerg Med 22:182-191

15. Goffi A, Pivetta E, Lupia E et al (2013) Has lung ultrasound an impact on the management of patients with acute dyspnea in the emergency department? Crit Care 17(4):R180 
16. Yuan A, Yang PC, Chang YC et al (2001) Value of chest sonography in the diagnosis and management of acute chest disease. J Clin Ultrasound 29:78-86

17. Agarwal A, Barman B, Parihar A et al (2020) Impact of bedside combined cardiopulmonary ultrasound on etiological diagnosis and treatment of acute respiratory failure in critically ill patients. Indian J Crit Care Med 24:1062-1070

18. Haji K, Haji D, Canty DJ et al (2018) The feasibility and impact of routine combined limited transthoracic echocardiography and lung ultrasound on diagnosis and management of patients admitted to ICU: a prospective observational study. J Cardiothorac Vasc Anesth 32:354-360

19. Wallbridge PD, Joosten SA, Hannan LM et al (2017) A prospective cohort study of thoracic ultrasound in acute respiratory failure: the $\mathrm{C} 3 \mathrm{PO}$ protocol. JRSM Open 8:205427041769505

20. Xirouchaki N, Kondili E, Prinianakis G et al (2014) Impact of lung ultrasound on clinical decision making in critically ill patients. Intensive Care Med 40:57-65

21. Kröner A, Binnekade JM, Graat ME et al (2008) On-demand rather than daily-routine chest radiography prescription may change neither the number nor the impact of chest computed tomography and ultrasound studies in a multidisciplinary intensive care unit. Anesthesiology 108:40-45

22. Mozzini C, Di Dio PM, Pesce G et al (2018) Lung ultrasound in internal medicine efficiently drives the management of patients with heart failure and speeds up the discharge time. Intern Emerg Med 13:27-33

23. Sferrazza Papa GF, Mondoni M, Volpicelli G et al (2017) Point-of-care lung sonography: an audit of 1150 examinations. J Ultrasound Med 36:1687-1692

24. Yu CJ, Yang PC, Chang DB, Luh KT (1992) Diagnostic and therapeutic use of chest sonography: value in critically ill patients. Am J Roentgenol 159:695-701

25. Nakao S, Vaillancourt C, Taljaard M et al (2020) Evaluating the impact of point-of-care ultrasonography on patients with suspected acute heart failure or chronic obstructive pulmonary disease exacerbation in the emergency department: a prospective observational study. Can J Emerg Med 22:342-349

26. Koh Y, Chua MT, Ho WH et al (2018) Assessment of dyspneic patients in the emergency department using point-of-care lung and cardiac ultrasonography - a prospective observational study. J Thorac Dis 10:6221-6229

27. Silversides JA, Major E, Ferguson AJ et al (2017) Conservative fluid management or deresuscitation for patients with sepsis or acute respiratory distress syndrome following the resuscitation phase of critical illness: a systematic review and meta-analysis. Intensive Care Med 43:155-170

28. Bataille B, Riu B, Ferre F et al (2014) Integrated use of bedside lung ultrasound and echocardiography in acute respiratory failure: a prospective observational study in ICU. Chest 146:1586-1593

29. Pontet J, Yic C, Díaz-Gómez JL et al (2019) Impact of an ultrasound-driven diagnostic protocol at early intensive-care stay: a randomized-controlled trial. Ultrasound J 11(1):24

30. Zieleskiewicz L, Muller L, Lakhal K et al (2015) Point-of-care ultrasound in intensive care units: assessment of 1073 procedures in a multicentric, prospective, observational study. Intensive Care Med 41:1638-1647

31. Aakjær Andersen C, Brodersen J, Davidsen AS et al (2020) Use and impact of point-of-care ultrasonography in general practice: a prospective observational study. BMJ Open 10:e037664

32. Blaivas M, Kuhn W, Reynolds B, Brannam L (2005) Change in differential diagnosis and patient management with the use of portable ultrasound in a remote setting. Wilderness Environ Med 16:38-41

33. Scharonow M, Weilbach C (2018) Prehospital point-of-care emergency ultrasound: a cohort study. Scand J Trauma Resusc Emerg Med 26(1):49

34. Heldeweg MLA, Jagesar AR, Haaksma ME et al (2021) Effects of lung ultrasonography-guided management on cumulative fluid balance and other clinical outcomes: a systematic review. Ultrasound Med Biol 47:1163-1171

35. Wheeler AP, Bernard GR, Thompson BT et al (2006) Pulmonary-artery versus central venous catheter to guide treatment of acute lung injury. $N$ Engl J Med 354:2213-2224

36. Bailón MM, Rodrigo JMC, Lorenzo-Villalba N et al (2019) Effect of a therapeutic strategy guided by lung ultrasound on 6-month outcomes in patients with heart failure: Randomized, Multicenter Trial (EPICC Study). Cardiovasc Drugs Ther 33:453-459

37. Pang PS, Russell FM, Ehrman R et al (2021) Lung ultrasound-guided emergency department management of acute heart failure (BLUSHED-AHF): a Randomized Controlled Pilot Trial. JACC Heart Fail S2213-1779(21):00232-00238

38. Rusu DM, Siriopol I, Grigoras I et al (2019) Lung ultrasound guided fluid management protocol for the critically ill patient: study protocol for a multi-centre randomized controlled trial. Trials 20:236

\section{Publisher's Note}

Springer Nature remains neutral with regard to jurisdictional claims in published maps and institutional affiliations.

\section{Submit your manuscript to a SpringerOpen ${ }^{\circ}$ journal and benefit from:}

- Convenient online submission

- Rigorous peer review

- Open access: articles freely available online

- High visibility within the field

Retaining the copyright to your article

Submit your next manuscript at springeropen.com 